Bull. Korean Math. Soc. 52 (2015), No. 3, pp. 955-962

http://dx.doi.org/10.4134/BKMS.2015.52.3.955

\title{
$H$-TREES, RESTRICTIONS OF DOWLING GROUP GEOMETRIES
}

\author{
Eunice Mphako-Banda
}

\begin{abstract}
It has been established that the role played by complete graphs in graph theory is similar to the role Dowling group geometries and Projective geometries play in matroid theory. In this paper, we introduce a notion of $H$-tree, a class of representable matroids which play a similar role to trees in graph theory. Then we give some properties of $H$-trees such that when $q=0$, then the results reduce to the known properties of trees in graph theory. Finally we give explicit expressions of the characteristic polynomials of $H$-trees, $H$-cycles, $H$-fans and $H$-wheels.
\end{abstract}

\section{Introduction}

Matroid theory has been developed mainly out of a deep examination of the properties of independence and dimension in vector spaces. A second original source for the theory of matroids is graph theory. Hence matroid theory borrows extensively from the terminology of linear algebra and graph theory. One research area in matroid theory is to extend results from graph theory to matroids. The biggest challenge in this research area is that there is no real notion of a vertex for a matroid. Irrespective of this hurdle, some concepts of graphs have been extended successfully to matroids. For example, the notion of vertex-join has been extended to matroids as $q$-cones in projective geometries [8] and as $H$-lifts in Dowling geometries in [5]. Other concepts like Eurelian, bipartite, chromatic polynomial, Tutte polynomial just to mention a few have been defined for matroids, for which we refer the reader to $[2,4,7]$.

In graph theory, complete graphs and trees play a big role in the study of graphs. It has been established that the role played by complete graphs in graph theory is similar to the role Dowling group geometries and Projective geometries play in matroid theory. What do we know about classes of matroids playing a role similar to trees in graph theory? It has been established that a

Received June 10, 2014; Revised September 2, 2014

2010 Mathematics Subject Classification. Primary 05B35, 05C31; Secondary 05C05, $05 \mathrm{C} 07$.

Key words and phrases. Dowling group geometry, matroid, tree, characteristic polynomial.

Supported wholly by the National Research Foundation of South Africa, Grant no. 86330. 
rank $r$ uniform matroid is isomorphic to a cycle matroid of a tree with $r$ edges, but this does not answer our question.

In this paper, we investigate a class of representable matroids playing a role similar to trees in graph theory. We introduce a class of matroids called $H$ trees, give some properties of $H$-trees which reduces to known properties of trees in graph theory if $q=0$. Then we use the concept of $H$-trees to define $H$-cycles, $H$-fans and $H$-wheels. Finally, we derive explicit expressions of the characteristic polynomials of these matroids.

\section{Representable matroids}

We outline the properties of Dowling group geometries that we need in this paper. Then we define a representable matroid and some terminology to be used in the paper. For further details, we refer the reader to $[3,6]$.

A matroid $M(E)$ is a set $E$ with a rank function $r$, for which the following properties hold:

(i) If $X \subseteq E$, then $0 \leq r(X) \leq|X|$.

(ii) If $X \subseteq Y \subseteq E$, then $r(X) \leq r(Y)$.

(iii) If $X$ and $Y$ are subsets of $E$, then

$$
r(X \cup Y)+r(X \cap Y) \leq r(X)+r(Y) .
$$

A matroid can be defined by its set of independent sets, set of circuits, set of bases, rank function and closure operator. All these different ways of defining a matroid are equivalent. A rank- $r$ Dowling group geometry over a finite group $H$, denoted by $Q_{r}(H)$, has two kinds of points and three types of lines; coordinate points $b_{1}, b_{2}, \ldots, b_{r}$ which form a basis, these points are also known as the joints of $Q_{r}(H)$; non-coordinate points $h_{i j}$ for every $h \in H$ and every pair of indices $1 \leq i<j \leq r$ such that $h_{i j} \in \operatorname{cl}\left\{\mathrm{b}_{\mathrm{i}}, \mathrm{b}_{\mathrm{j}}\right\}$; coordinate lines $b_{i} \vee b_{j}=b_{i} \cup b_{j} \cup\left\{h_{i j} \mid h \in H\right\}$; transversal lines $\left\{h_{i j}, g_{j k},(h g)_{i k}\right\}$ for each pair $h, g \in H$ residing on intersecting coordinate lines and trivial lines connecting any two points not already on a common line. It is clear from this definition that a rank- $r$ Dowling group geometry over a finite group $H$ is a matroid. A matroid $M$ is representable over a group $H$ if $M$ is isomorphic to $Q_{r}(H) \mid S$ for some subset $S$.

Note. We will use the following terminology, for any matroid $M \cong Q_{r}(H) \mid S$, a coordinate line is said to be empty if it has no non-coordinate points, otherwise the coordinate line is said to be non-empty.

We will borrow some terminology from graph theory throughout this paper and define it for representable matroids.

Definition. The degree of a joint, $b_{i}$, of a representable matroid is the number of non-empty coordinate lines incident with the joint, denoted by $d\left(b_{i}\right)$. It is clear from this definition that the degree of each joint for a rank $r$ Dowling geometry is $r-1$. 


\section{3. $\boldsymbol{H}$-trees}

Trees are a class of graph which plays a central role in graph theory. In this section we mimick the definition and some properties of trees in graph theory for representable matroids. We will start by defining a complete $H$-tree and then we generalize to what we shall call an $H$-tree.

Definition. Let $Q_{r}(H)$ be a rank- $r$ Dowling group geometry over a finite group $H$ of order $q$, with joints $b_{1}, b_{2}, \ldots, b_{r}$. Let $r \in \mathbb{Z}$ such that $q \geq 1, r>1, u<r$ and let a rank-r matroid $M=Q_{r}(H) \mid S$ such that joints $b_{1}, b_{2}, \ldots, b_{u} \in S$. We define the matroid $M$ to be a complete $H$-tree if the following properties hold:

(i) there are $r-1$ non-empty coordinate lines with exactly $q+2$ points;

(ii) for each triple $\left\{b_{i}, b_{j}, b_{k}\right\} \subseteq\left\{b_{1}, b_{2}, \ldots, b_{r}\right\}$ at least one coordinate line is empty.

We denote a rank $r$ complete $H$-tree by $t_{r}^{q}(H)$, where $q$ is the order of group $H$. We define each non-empty coordinate line with $q+2$ elements to be an arm of the complete $H$-tree. From definition, it is clear that we have complete $H$-trees of rank $r$ which are not isomorphic. Recall that $h_{i j}$ represents the $q$ non-coordinate points on the coordinate line $b_{i} \vee b_{j}$. We define a complete $H$-star to be a complete $H$-tree, $t_{r}^{q}(H)$, with elements of the form $\left\{b_{1} \cup h_{12} \cup\right.$ $b_{2} \cup h_{13} \cup b_{3} \cup \cdots \cup h_{1 r} \cup b_{r}$ \}, denoted by $S_{r}^{q}(H)$. We define a complete $H$ path to be a complete $H$-tree, $t_{r}^{q}(H)$, of rank $r>2$ with elements of the form $\left\{b_{1} \cup h_{12} \cup b_{2} \cup h_{23} \cup b_{3} \cup h_{34} \cup \cdots \cup b_{r-1} \cup h_{(r-1) r} \cup b_{r}\right\}$, denoted by $P_{r}^{q}(H)$. Two diagrams in Figure 1, clarifies the definition of a complete $H$-tree of rank $r, t_{r}^{q}(H)$.
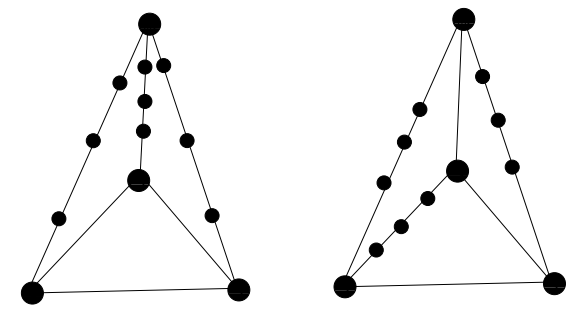

FiguRE 1

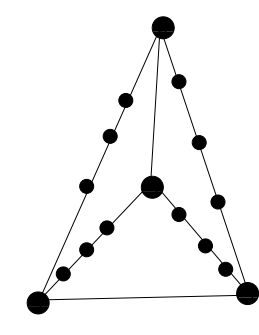


and shall be denoted by $t_{r}^{0}(H)$. Hence all our proposed theory should reduce to the known theory on trees in graph theory when $q=0$, because $t_{r}^{0}(H)$ is the cycle matroid of a tree on $r+1$ vertices.

Proposition 3.1. Let a matroid $M$ be a complete $H$-tree of rank $r$. Then

(a) $M$ has $r-1$ arms.

(b) $M$ has $r$ coordinate points.

(c) $M$ is a complete $H$-star if it has one joint of degree $r-1$ and all the other joints have degree one.

(d) $M$ is a complete $H$-path if it has two joints of degree one and all the other joints have degree two.

(e) $M$ is isomorphic to $U_{2, q+2}$ if $r=2$.

The following theorem is one of the basic properties of complete $H$-trees which reduces to a well known result in graph theory.

Theorem 3.2. Let a matroid $M=Q_{r}(H) \mid S$ be a complete $H$-tree of rank $r$. Then $M$ has exactly $1+(r-1)(q+1)$ elements.

Proof. The proof is by induction on the rank $r$ of the $H$-tree. Let the set of the $q$ non-coordinate points on the coordinate line $b_{i} \vee b_{j}$, be labelled as $\left\{h_{i j_{1}}, h_{i j_{2}}, \ldots, h_{i j_{q}}\right\}$. Let $M$ be a rank 2 complete $H$-tree. Then $M$ is isomorphic to the uniform matroid $U_{2, q+2}$, hence $M$ has $q+2$ points. Assume it is true for any rank $k H$-tree. Now we consider any rank $k+1 H$-tree. Let $M=Q_{k}(H) \mid S$ be a rank $k$ complete $H$-tree and let $M^{\prime}=Q_{k+1}(H) \mid S^{\prime}$ be a rank $k+1$ complete $H$-tree. $M^{\prime}$ can be constructed from $M$ by embedding the matroid $M$ in $Q_{k+1}(H)$ and adding one more arm to $M$. Choose any one joint $b_{i} \in S$, and define the set

$$
S^{\prime}=S \cup b_{k+1} \cup\left\{h_{i(k+1)_{1}}, h_{i(k+1)_{2}}, \ldots, h_{i(k+1)_{q}}\right\},
$$

where the joint $b_{k+1} \in Q_{k+1}(H)$ but $b_{k+1} \notin Q_{k}(H)$. Clearly, the constructed matroid $M^{\prime}=Q_{k+1}(H) \mid S^{\prime}$ is a complete $H$-tree of rank $k+1$ by definition. The number of elements of $M^{\prime}$ is

$$
\left|S^{\prime}\right|=|S|+\left|\left\{b_{k+1}\right\}\right|+\left|\left\{h_{i(k+1)_{1}}, h_{i(k+1)_{2}}, \ldots, h_{i(k+1)_{q}}\right\}\right| .
$$

Hence by induction hypothesis we have

$$
\left|S^{\prime}\right|=1+(k-1)(q+1)+1+q=1+k q-q+k-1+1+q=1+k(q+1),
$$

thus proving our theorem.

The following corollary is a well known result in graph theory.

Corollary 3.3. Let the graph $t_{n}$ be a tree on $n$ vertices. Then $t_{n}$ has $n-1$ edges.

Proof. The cycle matroid of $t_{n}$ has rank $n-1$ and is isomorphic to $U_{n-1, n-1} \cong$ $t_{n-1}^{0}(H)$. Hence $E\left(t_{n}\right)=1+(n-1-1)(0+1) 1=n-1$ as required. 
Definition. In general a rank $r$ submatroid of a complete $H$-tree, $t_{r}^{q}(H)$, will be called an $H$-tree and will be denoted $t_{r}(H)$. Similarly we have an $H$-star denoted $S_{r}(H)$ and an $H$-path denoted $P_{r}(H)$.

\section{Characteristic polynomial of an $\boldsymbol{H}$-tree}

The characteristic polynomial of a tree, has a very simple explicit formula. In this section we give the characteristic polynomial of a complete $H$-tree and show that it reduces to the characteristic polynomial of a tree in graph theory when $q=0$. [1].

To prove our next result we need the following known Theorem 4.1 found in

Theorem 4.1. Let $F$ be a modular hyperplane of a matroid $M$ and let $C^{*}=$ $E(M)-F$. Then

$$
\chi(M ; \lambda)=\left(\lambda-\left|C^{*}\right|\right) \chi(M \backslash F ; \lambda) .
$$

Theorem 4.2. The characteristic polynomial of a complete $H$-star, $S_{r}^{q}(H)$ is

$$
\chi\left(S_{r}^{q}(H) ; \lambda\right)=(\lambda-1)(\lambda-(q+1))^{r-1} .
$$

Proof. It is clear that a complete $H$-star, $S_{r}^{q}(H)$, has a modular hyperplane isomorphic to $S_{r-1}^{q}(H)$. The proof by induction on the number of arms of a complete $H$-star is straightforward.

The following result shows that all non-isomorphic complete $H$-trees are $\chi$ equivalent and when $q=0$ it reduces to a well known result in graph theory that all non-isomorphic trees are $\chi$ - equivalent.

Theorem 4.3. Let a matroid $M=Q_{r}(H) \mid S$ be a complete $H$-tree $t_{r}^{q}(H)$. Then the characteristic polynomial of $M$ is

$$
\chi(M ; \lambda)=(\lambda-1)(\lambda-(q+1))^{r-1} .
$$

Proof. Let $M=Q_{r}(H) \mid S=t_{r}^{q}(H)$, where $q$ is the order of group $H$ and $r$ is the rank of $M$. By definition it is clear that a complete $H$-tree of any rank $r$ has $r-1$ arms and $r$ joints. It is also clear that non-isomorphic complete $H$-trees have different degree sequences. We show that we can construct $\chi$ - equivalent non-isomorphic complete $H$-trees of any rank $r$. The proof is by induction on $n$, the number of joints of $\operatorname{deg}>1$ of $M$. When $n=1$ and any rank $r \geq 2$, we have a special complete $H$-tree called a complete $H$-star and by Theorem 4.2 the result is true.

We assume it is true when $n=k<r-2$ and any rank $r \geq 2$. We now construct a rank $r$ complete $H$-tree with $k+1$ joints of deg $>1$. Let $b_{1}, b_{2}, \ldots, b_{k}$ be the $k$ joints of deg $>1$ in a rank $r-1$ complete $H$-tree, $t_{r-1}^{q}(H)$. Without loss of generality, consider that the coordinate line $b_{k-1} \vee b_{k}$ is an arm of the $H$-tree, $t_{r-1}^{q}(H)$. There are $q+2$ points on this line. Now, add another joint $b_{k+1}$ and the $q$ points, $h_{k, k+1_{1}}, h_{k, k+1_{2}}, \ldots, h_{k, k+1_{q}}$ so that the coordinate line $b_{k} \vee b_{k+1}$ have $q+2$ points. Since the joint $b_{k+1} \notin t_{r-1}^{q}(H)$, by definition, we 
have constructed a rank $r, H$-tree, $t_{r}^{q}(H)$. From this construction it is clear that $t_{r}^{q}(H) \backslash\left\{h_{k, k+1_{1}}, h_{k, k+1_{2}}, \ldots, h_{k, k+1_{q}}\right\}$ is isomorphic to $t_{r-1}^{q}(H)$. Moreover $t_{r}^{q}(H) \backslash\left\{h_{k, k+1_{1}}, h_{k, k+1_{2}}, \ldots, h_{k, k+1_{q}}\right\}$ is a modular hyperplane of $t_{r}^{q}(H)$. Hence by applying Theorem 4.1 and induction assumption, we have

$$
\begin{aligned}
\chi\left(t_{r}^{q}(H) ; \lambda\right) & =(\lambda-(q+1)) \chi\left(t_{r}^{q}(H) \backslash\left\{h_{k, k+1_{1}}, h_{k, k+1_{2}}, \ldots, h_{k, k+1_{q}}\right\} ; \lambda\right) \\
& =(\lambda-(q+1)) \chi\left(t_{r-1}^{q}(H) ; \lambda\right) \\
& =(\lambda-(q+1))(\lambda-1)(\lambda-(q+1))^{r-2} \\
& =(\lambda-1)(\lambda-(q+1))^{r-1} .
\end{aligned}
$$

By repeated application of the construction in this proof, we can construct a complete $H$-tree of any degree sequence possible, from a rank 2 complete $H$ tree. Hence by induction, the theorem is true for any complete $H$-tree $t_{r}^{q}(H)$ with any degree sequence.

Corollary 4.4. The characteristic polynomial of a graph $t_{n}$ is $(\lambda-1)^{n-1}$.

\section{Characteristic polynomial of an $\boldsymbol{H}$-cycle}

In this section we define an $H$-cycle based on the definition of an $H$-tree. We then give some properties and an explicit expression of the characteristic polynomial of an $H$-cycle.

Definition. We define a complete $H$-cycle of rank $r>2$ to be a matroid with elements of the form $\left\{b_{1} \cup h_{12} \cup b_{2} \cup h_{23} \cup b_{3} \cup h_{34} \cup \cdots \cup b_{r-1} \cup h_{(r-1) r} \cup b_{r} \cup\right.$ $\left.h_{1 r}\right\}$, denoted by $C_{r}^{q}(H)$, i.e., it is formed by adding the non-coordinate points between the two joints of degree one of an $H$-path.

An example of a complete $H$-cycle, $C_{r}^{q}(H)$ is shown in Figure 1. If a nonempty coordinate line is added to a rank $r H$-tree, an $H$-cycle is created.

Proposition 5.1. Let a matroid $M$ be a complete $H$-cycle of rank $r$. Then

(a) $M$ has $r$ arms.

(b) $M$ has $r$ coordinate points.

(c) all the joints have degree 2.

(d) deletion of one arm results into an $H$-path.

We need the following theorem given in [4] to prove our next result.

Theorem 5.2. Let $Q_{r}(H)$ be a rank-r Dowling group geometry over a finite group $H$ of order $q$. Then the characteristic polynomial of $Q_{r}(H)$ is

$$
\chi\left(Q_{r}(H) ; \lambda\right)=(\lambda-1)(\lambda-q-1)(\lambda-2 q-1) \cdots(\lambda-(r-1) q-1) .
$$

Theorem 5.3. Let a matroid $M=Q_{r}(H) \mid S$ be a complete Dowling cycle $C_{r}^{q}(H)$ and $r \geq 3$. Then the characteristic polynomial of $M$ is

$$
\chi(M ; \lambda)=(\lambda-q-1)^{r}+(-1)^{r} q^{r-1}(\lambda-1) .
$$


Proof. The proof is by induction on $r$. Let $r=3$, then $C_{3}^{q}(H)=Q_{3}(H)$. Hence by Theorem 5.2 , we get

$$
\begin{aligned}
\chi\left(C_{3}^{q}(H) ; \lambda\right) & =\chi\left(Q_{3}(H) ; \lambda\right) \\
& =(\lambda-1)(\lambda-q-1)(\lambda-2 q-1) \\
& =(\lambda-q-1)^{3}-q^{2}(\lambda-1) .
\end{aligned}
$$

Therefore the result is true for $r=3$. Assume it is true for some $r=k$. Now consider $C_{k+1}^{q}(H)$. By repeated application of the deletion and contraction formula and applying Theorem 4.3 and the induction assumption, we get

$$
\begin{aligned}
\chi\left(C_{k+1}^{q}(H) ; \lambda\right) & =\chi\left(t_{k+1}^{q}(H) ; \lambda\right)-q \chi\left(C_{k}^{q}(H) ; \lambda\right) \\
& =(\lambda-1)(\lambda-(q+1))^{k+1}-q\left[(\lambda-q-1)^{k}+(-1)^{k} q^{k-1}(\lambda-1)\right] \\
& =(\lambda-1)(\lambda-(q+1))^{k}[(\lambda-q-1)]+(-q)(-1)^{k} q^{k-1}(\lambda-1) \\
& =(\lambda-q-1)^{k+1}+(-1)^{k+1} q^{k}(\lambda-1) .
\end{aligned}
$$

The result is true for any complete $H$-cycle $C_{r}^{q}(H)$.

Note that when $q=0$ for a complete $H$-cycle $C_{r}^{q}(H)$, we get $U_{r, r}$ and this verifies the characteristic polynomial of a graph $t_{n}$ is $(\lambda-1)^{n-1}$.

\section{Characteristic polynomials of an $H$-fan and an $H$-wheel}

In this section we define an $H$-fan and an $H$-wheel and we give explicit expressions of the characteristic polynomials of these matroids. An operation similar to a vertex-join of a graph was defined for representable matroids in [5] as an $H$-lift.

Definition ([5]). Let $\left\{b_{1}, b_{2}, \ldots, b_{r-1}\right\}$ be the set of joints of $Q_{r}(H)$. Let $A \subseteq$ $\operatorname{cl}\left\{b_{1}, b_{2}, \ldots, b_{r-1}\right\}$. An $H$-lift in $Q_{r}(H)$ is the matroid $Q_{r}(H) \mid A \cup\left\{b_{r}\right\} \cup h_{i r}$ : $h \in H, 1 \leq i \leq r-1$. The set $A$ is called the base of the $H$-lift.

We define a rank- $r H$-fan to be an $H$-lift of a rank- $(r-1) H$-path and is denoted by $F_{r}^{q}(H)$. We define a rank- $r H$-wheel to be an $H$-lift of a rank$(r-1) H$-cycle and is denoted by $W_{r}^{q}(H)$. We need the following theorem before stating our next results.

Theorem 6.1 ([5]). Let the matroid $M$ be an $H$-lift with base $A$. Then the characteristic polynomial of $M$ is

$$
\chi(M ; \lambda)=(\lambda-1) \chi(A ; \lambda-q+1) .
$$

The following results are derived by applying Theorem 4.3, Theorem 5.3, Theorem 6.1 and the definitions.

Theorem 6.2. The characteristic polynomial of $F_{r}^{q}(H)$, an $H$-fan of rank $r$ is

$$
\chi\left(F_{r}^{q}(H) ; \lambda\right)=(\lambda-1)(\lambda-q)(\lambda-2 q)^{r-2} .
$$


Theorem 6.3. The characteristic polynomial of $W_{r}^{q}(H)$, an $H$-wheel of rank $r$ is

$$
\chi\left(W_{r}^{q}(H) ; \lambda\right)=(\lambda-1)\left[(\lambda-2 q)^{r-1}+(-1)^{r-1} q^{r-2}(\lambda-q)\right] .
$$

\section{References}

[1] T. H. Brylawski, Modular constructions for combinatorial geometries, Trans. Amer. Math. Soc. 203 (1975), 1-44.

[2] H. H. Crapo, The Tutte polynomial, Aequationes Math. 3 (1969), 211-229.

[3] T. A. Dowling, A class of geometric lattices based on finite groups, J. Combin. Theory Ser. B. 14 (1973), 61-86. erratum, J. Combin. Theory Ser. B. 15 (1973), 211.

[4] J. P. S. Kung, Critical problems, Matroid theory (Seattle, WA, 1995), 1-127, Contemp. Math., 197, Amer. Math. Soc., Providence, RI, 1996.

[5] E. G. Mphako, H-lifts of tangential k-blocks, Discrete Math. 285 (2004), no. 1-3, 201-210.

[6] J. G. Oxley, Matroid Theory, Oxford University Press, New York, 1992.

[7] D. J. A. Welsh, Euler and bipartite matroids, J. Combin. Theory 6 (1969), no. 4, 375-377.

[8] G. P. Whittle, q-lifts of tangential k-blocks, J. London Math. Soc. (2) 39 (1989), no. 1, $9-15$.

The John Knopfmacher Centre for Applicable Analysis and Number Theory School of Mathematics

UNIVERSiTY OF THE WitWATERSRAND

P/Bag 3, Wits, 2050, Republic of South Africa

E-mail address: Eunice.Mphako-Banda@wits.ac.za 